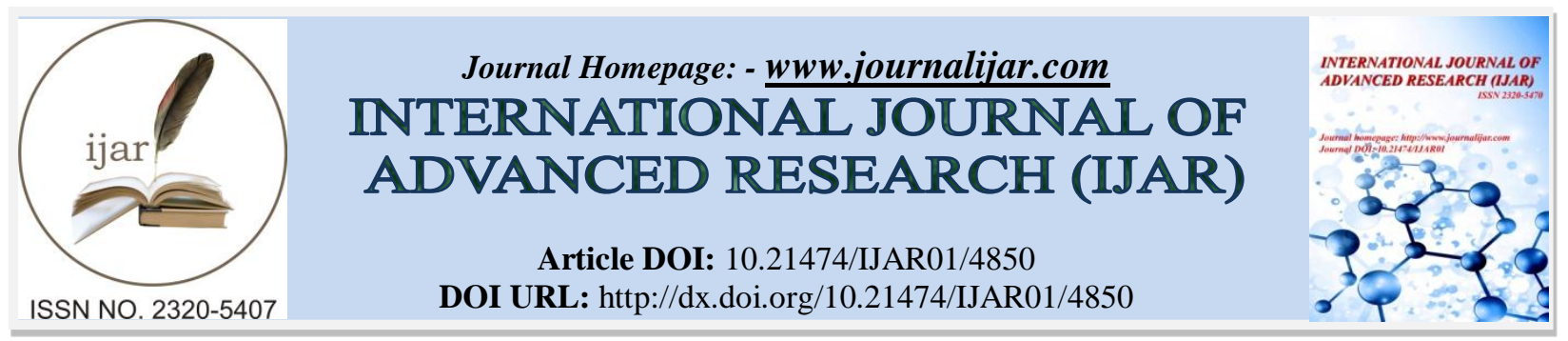

RESEARCH ARTICLE

\title{
EFFECT OF WATCHING TELEVISION ON VISION OF SCHOOL CHILDREN IN MADURAI.
}

Dr. R. Priyadarsini, Dr. L. Santhanalakshmi, Dr. K. Kanchana and Dr. P. Shanmugapriya. Institute of Physiology, Madurai Medical College, Madurai.

\section{Manuscript Info}

Manuscript History

Received: 14 May 2017

Final Accepted: 16 June 2017

Published: July 2017

Key words:-

Refractive error, prevalence, school

children, watching television

\section{Abstract}

Background: Refractive errors are one of the major causes of correctable blindness.

Aim: To find the association between watching television and vision of school children aged 10-14 years.

Methods: A cross sectional school based study with 600 students of age 10-14 years. Prior informed consent for participation in the study was obtained from their parents. Visual acuity was tested using Snellen's E chart. Using retinoscopy and subjective refraction, refractive error was categorized as myopia, hypermetropia and astigmatism A standardized questionnaire prepared in their local language was used to collect data on their age, sex and duration of watching television. Data was analyzed by Pearson's Chi Square test using SPSS software.

Results: Prevalence of refractive errors in school children was 16.2 $\%$. Watching television for more than five years was significantly associated with development of refractive errors. $(\mathrm{p}=0.009)$

Conclusion: A longitudinal follow up of all the children with analysis of various risk factors may be required to give a clearer picture.

Copy Right, IJAR, 2017,. All rights reserved.

\section{Introduction:-}

The quality of life of any person depends on many aspects. One's ability to see things clearly can determine success or failure at work or in school. Normal vision helps a person to lead an independent life, without having to rely on others to fulfill their basic needs.

It has been estimated that about 180 million people worldwide are visually disabled. 45 million of them are blind. Developing countries account for four out of five of the visually disabled. One of the major causes of correctable blindness is refractive error. It has been estimated that refractive errors are prevalent in about 2.3 billion people. Out of these, 1.8 billion people have access for examination of the eyes and proper treatment. The remaining 500 million people have uncorrected refractive errors resulting in impaired vision and blindness.

Refractive errors are the commonest cause of impaired vision and they form the second important cause of treatable blindness in the world next only to cataract.

Refractive error is an intrinsic optical defect where parallel light rays are not focussed on the retina, resulting in a blurred image.Refractive errors develop during childhood and adolescence. If they are not treated some children may develop amblyopia or strabismus. 
Refractive errors may not only be associated with genetic factors but also with various other risk factors like prolonged near activity like reading, playing video games and watching television or computer for longer duration which considerably reduces the outdoor play time of children. Hence this study has been undertaken to study the prevalence of refractive errors in school children and to find their association with hereditary and environmental factors. It is expected that this study will also give an insight into the magnitude of this problem and create awareness among the common public.

\section{Aim And Objectives:-}

1. To find the prevalence of refractive errors in school children aged 10-14 years.

2. To study the association between watching television and refractive errors.

\section{Refractive Errors:-}

A condition of refractive error ametropia is defined as the state of refraction wherein the parallel rays of light coming from infinity are focussed either in front or behind the light sensitive layer of the retina in one or both meridian with accommodation at rest.

Ametropia includes

$>$ Myopia

$>$ Hypermetropia

$>$ Astigmatism.

\section{Measurement of visual acuity:-}

In clinical practice, measurement of visual acuity is synonymous with the measurement of minimum resolvable. The threshold of the minimum resolvable is between 30 seconds and 1 minute of arc. Hence, all clinical tests employed to measure visual acuity are designed taking into consideration the threshold of the one minimum resolvable.

Snellen's E chart test is one of the commonly used acuity tests. The basis of Snellen's test types is that two distant points can be visible as separate only when they subtend an angle of 1 minute at the nodal point of the eye.

It consists of a series of black capital letters on a white board arranged in lines each progressively diminishing in size. The lines comprising the letters have such a breadth that they will subtend an angle of 1 minute at the nodal point of the eye.

Each letter of the chart is so designed that it fits in a square, the sides of which are five times the breadth of the constituent line. At a particular distance each letter subtends an angle of 5 minutes at the nodal point of the eye.

\section{Procedure of Testing:-}

For testing distant visual acuity, the individual is seated at a distance of 6 meters from the Snellen's chart, so that the rays of light are practically parallel and the person exerts minimal accommodation. The chart should be adequately illuminated with not less than 20 foot candles. The person is asked to read the chart with each eye separately and the visual acuity is recorded as a fraction, the numerator being the distance of the person from the chart and the denominator being the smallest letters accurately read.

When the person is able to read the 6-meter line, the visual acuity is recorded as $6 / 6$, which is normal. Similarly, according to the smallest line the person can read from a distance of 6meters, the vision is recorded as 6/9,6/12, $6 / 18,6 / 24,6 / 36$ and $6 / 60$.

If one cannot read the top most line from 6meter, he is asked to walk slowly towards the chart, till one can read the top line. Depending on the distance from which one can read the top line vision is recorded as $5 / 60,4 / 60,3 / 60$, $2 / 60$, and $1 / 60$.

If the person is not able to read the top line even from 1meter, he is asked to count the fingers of the examiner. Vision is recorded as CF CF (counting fingers close to face). If one can appreciate only hand movements, visual acuity is recorded as HM positive.

When one cannot distinguish hand movements, perception of light is recorded and the acuity is recorded as PL positive or PL negative. 


\section{Retinoscopy:-}

Refractive errors can be diagnosed using the principle of retinoscopy. Cycloplegics like 1\% Tropicamide are used to produce ciliary muscle paralysis and pupillary dilatation.

The procedure is conducted in a dark room. The examiner sits 1 meter from the subject who wears a trial frame and fixes his gaze at a spot of light at a distance. A light is placed behind and above the subject's head. The examiner uses a plane mirror with a central hole to reflect the light into the subject's eyes. This produces a red reflex in the eye.

As the mirror is tilted from side to side, light is reflected into the eye and the direction of movement of the red reflex is noted.

\section{The results are interpreted as Follows:-}

1. If the shadow swirls around during movement of the mirror, the person is astigmatic.

2. No movement of red reflex- indicates myopia of $1 \mathrm{D}$

3. Movement of red reflex along with the movement of the mirror- indicates emmetropia, hypermetropia or myopia of less than $1 \mathrm{D}$.

4. Movement of red reflex against the movement of the mirror- indicates myopia of more than 1D.

5. Appropriate lenses are added to neutralize the red reflex from which the type of refractive error can be ascertained

\section{Materials and Methods:-}

A cross sectional school based study was designed with 600 students within the age group of 10-14 years from four schools ,selected randomly.

The principals, teachers and the concerned students were briefed about the study. Prior informed consent for participation in the study was obtained from the parents of these children.

A standardized questionnaire prepared in their local language was used to collect data on their age, sex, parental history of wearing glasses and other environmental factors like watching television, playing video games, outdoor activities and reading books.

Visual acuity (VA) test was performed using Snellen's E chart. Students with visual acuity $<6 / 6$ in one or both the eyes were tested for the presence or absence of refractive error by pinhole test. Those showing improvement with pinhole (indicating refractive error) were then subjected to retinoscopy and subjective refraction.

Emmetropia was defined as a spherical equivalent between -0.50 DS and +0.50 DS. A spherical equivalent less than -0.50 DS was taken as myopia. Hypermetropia was defined as a spherical equivalent greater than +0.50 DS. Astigmatism was defined as a cylindrical error less than -0.50 diopter cylinder (DC) in any axis.

Those children with both spherical and cylindrical errors were documented as having the spherical equivalent of myopia or hypermetropia respectively. The children with only cylindrical errors were documented as having astigmatism. Refractive error was categorized into three groups as i) myopia ii) hypermetropia and iii) astigmatism.

The particulars of each student were filled on a structured questionnaire specially designed for this purpose.

The data collected was analyzed by Pearson's Chi Square test using SPSS (Statistical Package for Social Sciences).

\section{Observation And Result:-}

Mean age of the 600 children examined was 12.05 years. Out of these children, 297 were boys and 303 were girls.

The results of the analysis are presented in the following table.

Prevalence of refractive errors in school children.

\begin{tabular}{|l|c|c|}
\hline & Number of children (600) & Percentage \\
\hline Refractive errors & 97 & 16.2 \\
\hline No refractive errors & 503 & 83.8 \\
\hline
\end{tabular}


The over-all prevalence of refractive errors in school children was found to be $16.2 \%$. Distribution of various types of refractive errors in school children.

\begin{tabular}{|l|c|c|}
\hline Type of refractive error & Number of children & Percentage \\
\hline Myopia & 67 & $69.1 \%$ \\
\hline Hypermetropia & 10 & $10.3 \%$ \\
\hline Astigmatism & 20 & $20.6 \%$ \\
\hline
\end{tabular}

Prevalence of refractive errors in school children watching television for years.

\begin{tabular}{|l|c|c|c|}
\hline Duration & $<5$ years & $>5$ years & p value \\
\hline Total & 287 & 313 & \\
\cline { 1 - 3 } Refractive error & $32[11.1 \%]$ & $65[20.8 \%]$ & $\mathbf{0 . 0 0 9}$ \\
\hline
\end{tabular}

When comparing the prevalence of refractive errors in children, depending on the years of watching television, there was a significant increase in the prevalence of refractive errors in children watching television for more than five years ( $20.8 \%$ ), compared to those children watching television for less than five years $(11.1 \%)$ with a $\mathrm{p}$ value of 0.009 .

Prevalence of refractive errors in children watching television (depending on the hours of watching television per day for the past one year)

\begin{tabular}{|l|c|c|c|}
\hline Duration & $<2$ (hours/day) & $>2$ (hours/day) & p value \\
\hline Total & 343 & 257 & \multirow{2}{*}{0.722} \\
\hline Refractive error & $53[15.5 \%]$ & $44[17.1 \%]$ & 0.7 \\
\hline
\end{tabular}

The prevalence of refractive errors in children who watched television for more than two hours per day was higher (17.1\%) compared to those who watched television for less than two hours per day (15.5\%), though it was not statistically significant.

\section{Discussion:-}

* School children form a vulnerable group affected by visual impairment. Murthy GVS, Gupta SK, et al., have reported in their study that $81.7 \%$ visual impairment in school age children had been due to refractive error.

* Both the children and their parents fail to recognize the vague symptoms produced by the presence of refractive errors. This creates subsequent detrimental effects on the educational potential, learning ability and the mental health of children.

* Once these children leave school, the chances of getting themselves screened for vision is reduced. If diagnosed, refractive error can be treated safely and effectively by simple methods. Hence this study was aimed at school children aged 10-14 years.

* In this present study, 97 out of the 600 school children had refractive errors. Myopia was found to be the commonest error being followed by astigmatism. Hypermetropia was found to be the least common error.

* This result is in accordance with the results obtained by Saw SM et al., 2001. This may be attributed to a change in life style and improved socioeconomic conditions with increased duration of near work, better education facilities, television and computer usage and reduction in outdoor activities.

* Studies by Afghani T et al., 2003, have showed that increasing literacy standards was associated with an increase in prevalence of refractive errors.

* Ayub A et al, 2007 found out in his study that there was an increase in prevalence of refractive errors in private sector schools, compared to government sector schools due to presence of the above said similar reasons.

* Myopia was found to be the commonest refractive error in our study being $\mathbf{6 9 . 0 9 \%}$ of the total. Astigmatism was the next common error forming $\mathbf{2 0 . 6 2 \%}$ of the total. Hypermetropia was the least common of all the errors contributing $\mathbf{1 0 . 3 1 \%}$ of the total.

* This is in accordance with the previous studies conducted on this subject by Hussain AB, Ahmed EK .The prevalence of refractive errors in school age children was $25.32 \%$ in their study. They also reported myopia in $63.5 \%$, hypermetropia in $11.2 \%$ and astigmatism in $20.4 \%$ children.

* Study of Junaid SM et al, shows an overall refractive error of $10 \%$ in school children. Hypermetropia was present in 58\% while myopia was present in 36\% children. Astigmatism was identified in $6 \%$ children. However their study reported a higher prevalence of hypermetropia than myopia which is contradictory to this present study. 
* Another study carried out by Ayub A et al demonstrated myopia in 43\%, hypermetropia in $21.5 \%$ and astigmatism in $35.5 \%$ children.

This study has also revealed an increase in the prevalence of refractive errors in children watching television for more number of years, which is at par with the Singapore- China study by Tan et al., 2002. Myopes demonstrated a positive association between the prevalence and duration of watching television- both in terms of years and hours per day.

\section{Conclusion:-}

This study has confirmed the finding of others that there is an increase in the prevalence of refractive errors in children of urban areas compared to those in rural areas. Myopia was found to be the commonest error being followed by astigmatism. Hypermetropia was found to be the least common error.

Environmental factors like watching television for more number of years contributed significantly to the development of refractive errors with a detrimental effect on the learning abilities and personality development of young children. With the advent of modern gadgets and improvement in technology, children are being constantly exposed more and more to the ill effects of these inventions. With increasing importance being given to academic activities and a reduction in duration of outdoor activities, due precautions must be taken by parents to ensure adequate visual hygiene in children.

Awareness on refractive errors and the importance of wearing glasses in children with refractive errors must be imparted in schools.

During the last few years, steps have been taken by the government to screen children in schools for early identification of refractive errors and their effective management by provision of spectacles.

Adequate screening procedures in schools, maintenance of visual hygiene and providing corrective measures will definitely reduce the burden of this problem in our society.

\section{Bibliography:-}

1. Murthy GVS. Vision testing for refractive Errors in schools. J. Comm. Eye health 2000; 3(33):3-4.

2. 2) Saw SM, Wu HM, Seet B, et al. Academic achievement, close up work parameters, and myopia in Singapore military conscripts. Br. J. Ophthalmol.2001; 85:855-860.

3. Afghani T, Vine HA, Bhatti A, Qadir M, Akhtar J, Tehzib M, Al- Shifa-Al-Noor (ASAN) refractive error study of one million school children. Pakistan J. Ophthalmol. 2003 Oct; 19 (4):101-7.

4. Ayub A, Imran A, Saima A. Prevalence of undetected refractive errors among school children. Biomedica. JulDec 2007; 23: 96-101.

5. Hussein AB, Ahmed EK. Refractive Errors in School Children of Tafila City. Rawal. Med. J. 2008; 33:85-87.

6. Junaid SM, Sethi S, Iqbal R. Frequency of Refractive errors in children attending eye out patient department agency head quarters hospital of LaudiKotal. G.J.M.S. July-Dec 2009; (7):2. 